\title{
Benign pigmented skin lesions
}

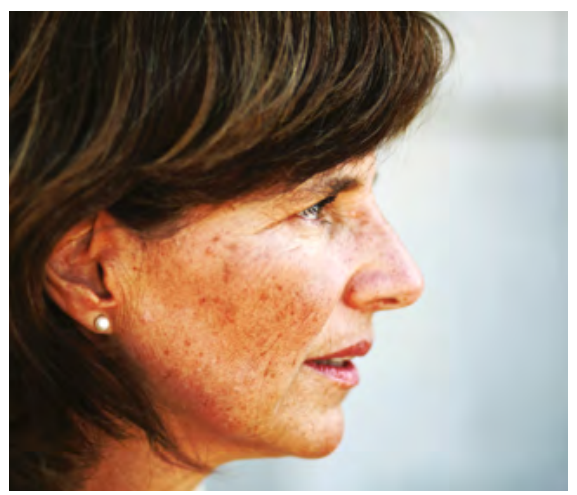

\section{CPD}

Philip Clarke

\section{Background}

Benign pigmented skin lesions are extremely common. Such lesions are seen every day in general practice.

\section{Objectives}

The objectives of this paper are to develop a framework that may be used to evaluate pigmented skin lesions and a strategy for dealing with pigmented lesions, outline the conditions that improve the diagnosis of pigmented lesions (eg good lighting, careful inspection and dermoscopy), and increase clinician confidence in identifying pigmented lesions with concerning features.

\section{Discussion}

Regular assessment of pigmented skin lesions during patient consultations, including in an opportunistic fashion, will increase diagnostic acumen and help to identify potentially problematic lesions, and may improve patient awareness of lesions on their skin.
PIGMENTED SKIN LESIONS are extremely common, with almost all patients having a number of pigmented lesions on their skin. When considering their various characteristics, it is useful to divide these lesions into melanocytic, keratinocytic, vascular and reactive lesions.

\section{Keratinocytic lesions}

\section{Seborrhoeic keratosis}

A very common lesion is the seborrhoeic keratosis (Figures 1-3). Seborrhoeic keratoses may start quite early in life, and it is not uncommon to find them on teenagers. They tend to become larger and more numerous with age. The pigment may vary from skin-coloured to pink, brown or black. Most seborrhoeic keratoses have a typical appearance and texture and characteristic dermoscopic features. ${ }^{1}$ However, care should be taken with any seborrhoeic keratosis that is unusual. Even very experienced clinicians are occasionally misled by such lesions, which may turn out to be a pigmented patch of Bowen's disease or a melanoma. If there is significant doubt about a seborrhoeic keratosis, the lesion should be biopsied or carefully reassessed in eight weeks, or the patient sent for a second opinion. Even though seborrhoeic keratoses are quite benign, malignancies do occasionally arise within a lesion Individual lesions may also become uncomfortable or unsightly, and treatment of such lesions is often appropriate.

\section{Basal cell carcinomas/Bowen's disease} Some basal cell carcinomas and in situ squamous cell carcinomas may be quite heavily pigmented, which may mask the diagnosis (Figures $2 \& 3$ ). Dermoscopy will usually reveal atypical blood vessels and characteristic pigment patterns to prompt the diagnosis. ${ }^{1}$

\section{Epidermal naevus}

This type of naevus is formed by overgrowth of one or more components of the epidermis. The lesions are often warty and hyperpigmented. They are often linear. Some examples include sebaceous naevus (often found on the scalp) and Becker naevus (usually around the shoulder). These are generally isolated abnormalities but are sometimes part of a congenital syndrome. This possibility needs to be kept in mind if there are other health issues for the child, or the naevus is extensive.

\section{Freckles and lentigines}

Freckles are also extremely common. Childhood freckles are induced by sun exposure and are more common in fair-skinned people, especially those with 
red hair. The colour of freckles is due to extra pigment induced by sun exposure, and not due to an increase in the number of melanocytes. Freckles often slowly reduce with age and sun protection. They tend to fade in the winter. Adult lentigines often appear later in life on the chronically exposed areas of the hands and face. These tend to be larger than childhood freckles. There is epidermal hyperplasia and an increase in the number of melanocytes. They tend not to fade in the winter. If any such lentigo on the face continues to grow and become irregular, lentigo maligna melanoma needs to be considered.

One very striking form of freckle is the inkspot. This appears as a very dark, irregular freckle on a sun damaged area of skin such as the shoulders or neck. Despite being very dark and irregular, it has a very typical appearance on dermoscopy, ${ }^{1}$ is quite stable and does not require removal if it remains stable.

Isolated melanocytic macules are quite common, especially on the lip. If there are several melanocytic macules appearing early in life, this may indicate an underlying syndrome. For instance, several macules on the lips and around and in the mouth may signify Peutz-Jeghers syndrome. Lifelong follow-up is required for such patients because of the extremely high likelihood of cancer, especially of the gastrointestinal tract.

\section{Melanocytic lesions}

Moles (melanocytic naevi) are also extremely common.

Congenital melanocytic naevi occur in approximately 1 in every 100 babies. $^{2}$ The risk of developing melanoma is very low, even in very large naevi. ${ }^{2}$

Most melanocytic naevi appear within the first few years of life, but new naevi may continue to appear throughout life. More naevi will develop if there is increased sun exposure, especially excessive exposure in the first 10 years of life. Excessive sun exposure in this time significantly increases the risk of melanoma later in life. ${ }^{3}$

Taking into consideration the number of naevi in the general population and the number of melanomas diagnosed, the risk of melanoma developing in any particular naevus is in the order of 1 in 10,000. ${ }^{2}$ Important clues to malignancy include unusual changes in colour or shape, and ongoing change in a mole.

Dysplastic naevi are difficult to accurately define, and a number of attempts have been made using clinical, histological and genetic criteria. However, a useful practical definition for general practice is an unusual-looking mole that is stable. Dysplastic naevi are not particularly more susceptible to malignant change, but they are a potentially important clue to overall melanoma risk. There is a significant correlation between having five or more dysplastic naevi and the lifetime risk of melanoma. ${ }^{3}$

Blue naevi are a distinctive form of melanocytic naevus. A blue naevus typically has a steely blue appearance with some characteristic features on dermoscopy. ${ }^{1}$ Malignant transformation is uncommon, but any change in a blue naevus should prompt careful evaluation.

The number of moles on a patient is also important. Having 100 or more moles relates to a significantly higher risk of melanoma. ${ }^{3}$ A very important phenotype to recognise in general practice is the patient with 100 or more moles, several dysplastic naevi and a family history of melanoma. These patients have a very high risk of melanoma and should be strongly advised to have a regular skin check. ${ }^{3}$

\section{Vascular lesions}

Haemangiomas are common in both children and adults (Figure 4). Childhood lesions usually clear spontaneously after a few years, but adult lesions persist and may become very numerous. Most are a very straightforward clinical diagnosis (eg cherry angioma), but some adult haemangiomas may be very dark and may present as a concerning black lesion. If the blood in the haemangioma becomes slow moving or thromboses, the very dark purple colour may appear black. There is a very characteristic appearance on dermoscopy. ${ }^{1}$

One uncommon but extremely important red lesion to be aware of is an amelanotic melanoma. These melanomas

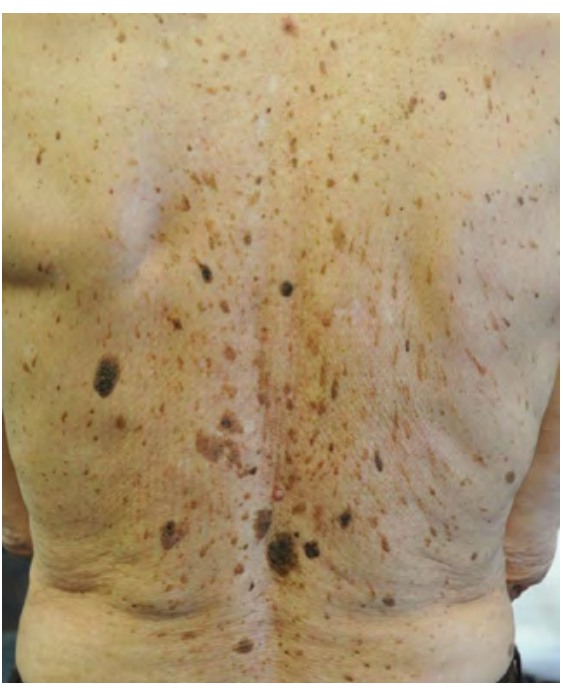

Figure 1. Multiple pigmented seborrhoeic keratoses

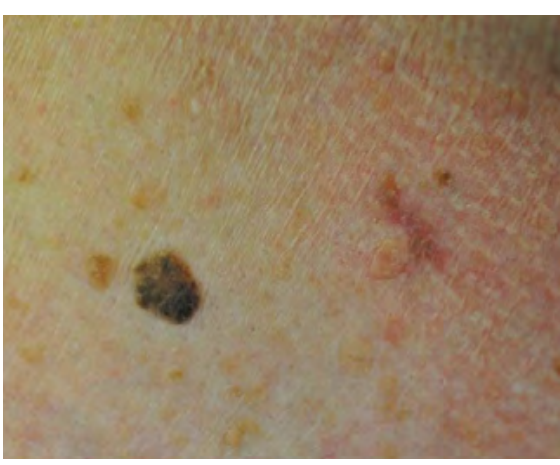

Figure 2. Pigmented seborrhoeic keratosis to the left and linear red basal cell carcinoma to the right

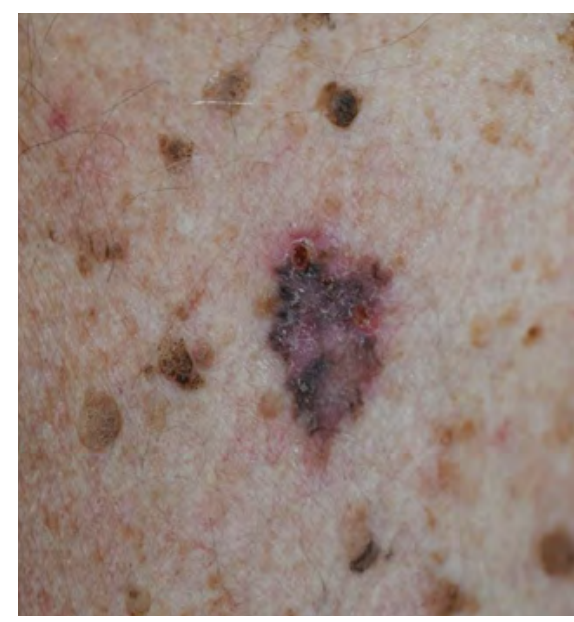

Figure 3. Pigmented basal cell carcinoma with surrounding pigmented seborrhoeic keratoses 
often have a very fast growth rate and have a poorer prognosis than most melanomas. Any firm red raised lesion that has persisted for four weeks should be biopsied. ${ }^{3}$ Such lesions may present as a presumed abscess, but if no pus is drained, the lesion should be biopsied (ie removed entirely).

Kaposi sarcoma is a vascular/lymphatic lesion that was rare prior to the increase in prevalence of human immunodeficiency virus. The diagnosis should be kept in mind when a vascular plaque or nodule is found.

\section{Reactive lesions}

\section{Dermatofibromas}

Dermatofibromas are quite common, especially on the lower limbs (Figure 5). The typical clinical finding is a very firm nodule that puckers when pressed from the sides. Some are quite deeply pigmented. They may vary in colour from skin-coloured to pink, red, violaceous or brown. Dermoscopy may show a typical pattern. ${ }^{1}$ Dermatofibromas are stable and benign, but rarely there can be malignant transformation (dermatofibrosarcoma prutuberans). If a lesion is enlarging, it should be removed.

\section{Eczema/lichen planus}

Small nodular patches of eczema may become quite pigmented. This is probably triggered by repeated scratching of the eczema. Lichen planus usually has a violaceous or brown pigmentation. Both conditions typically have lesions appearing in a symmetrical pattern, but careful attention should be taken with isolated lesions.

\section{Examination and dermoscopy}

Diagnosing pigmented lesions is enhanced by good lighting, a small amount of magnification (binocular loupe) and dermoscopy. Useful information is also obtained from feeling and stretching lesions. Rubbing lesions with an alcohol hand rub prior to dermoscopy is a useful way of highlighting vascular changes. Familiarity with dermoscopic findings and regular use of the dermatoscope are important skills for the general practitioner. $^{4}$

\section{Biopsy}

Most pigmented lesions will not need to be removed. However, if there is concern about the possibility of melanoma, it is important to remove the whole lesion with a thin margin $(2 \mathrm{~mm}) .{ }^{3}$ Punch and shave biopsies should not be performed on suspicious pigmented lesions. ${ }^{3}$ Histological interpretation and proper diagnosis is difficult, and malignant change may only be present in part of the lesion, which may be missed by partial biopsy.

\section{Key points}

- Benign pigmented skin lesions are extremely common in general practice and will be seen every day during consultations.

- A quick but careful examination aided by dermoscopy will rule out almost all serious lesions.

- Care needs to be taken with lesions that are not completely typical.

- It is important to take note of a patient's concern about a particular lesion, especially if it is an ongoing concern; such concerns should not be ignored or dismissed.

\section{Author}

Philip Clarke BMEDSc, MBBS, FRACGP, DFM, DDSc, FAAD, Dermatologist, Senior Clinical Lecturer, Department of Medicine, University of Tasmania, Tas Visiting Dermatologist, Launceston General Hospital, Tas.c17clarke@gmail.com

Competing interests: None.

Funding: None.

Provenance and peer review: Commissioned, externally peer reviewed.

\section{References}

1. Braun, RP, Rabinovitz HS, Oliviero M, Kopf AW, Saurat JH. Dermoscopy of pigmented skin lesions. J Am Acad Dermatol 2005;52(1):109-21. doi: 10.1016/j.jaad.2001.11.001.

2. Alikhan A, Ibrahimi OA, Eisen, DB. Congenital melanocytic nevi: Where are we now? Part I. Clinical presentation, epidemiology, pathogenesis, histology, malignant transformation, and neurocutaneous melanosis. J Am Acad Dermatol 2012;67(4):495.e1-17. doi: 10.1016/j. jaad.2012.06.023.

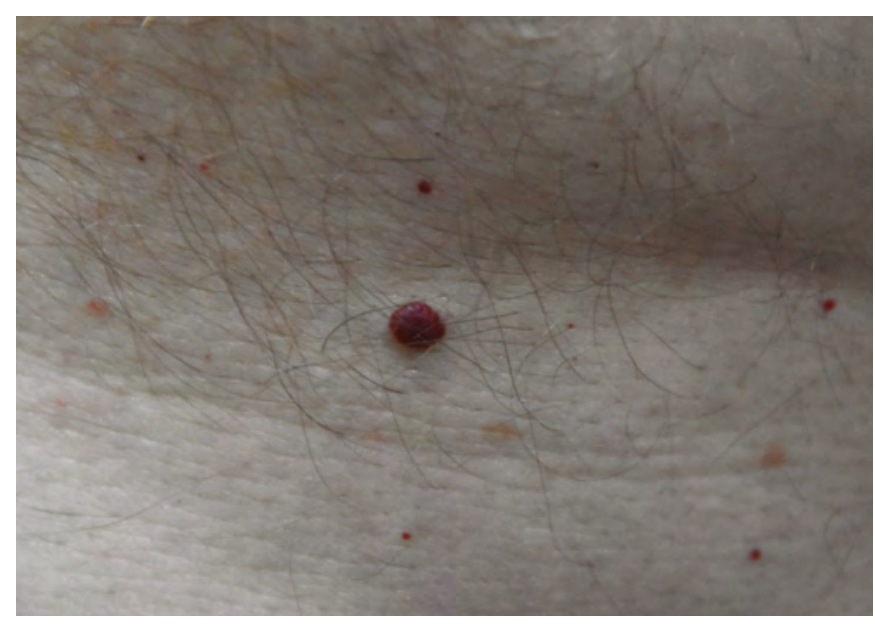

Figure 4. Dark capillary haemangioma

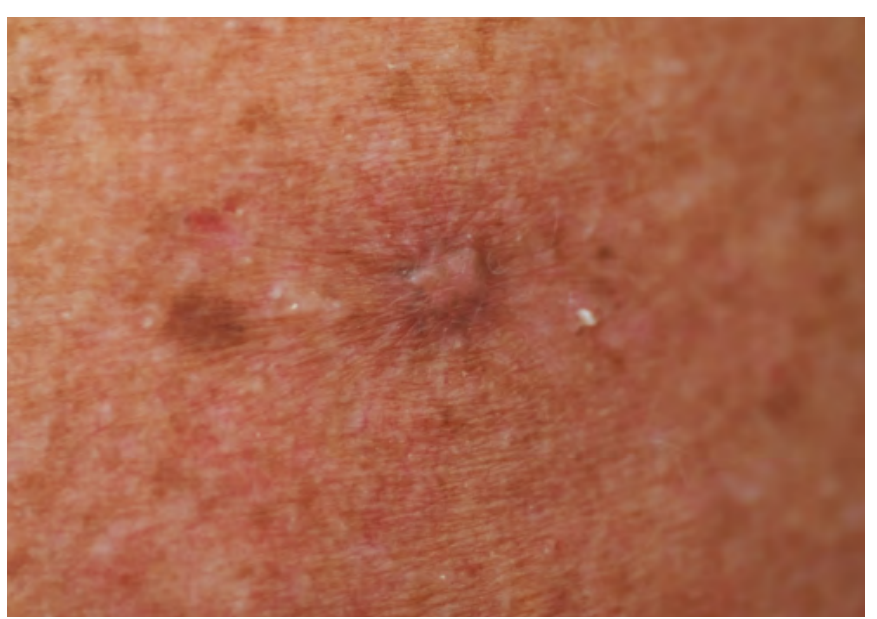

Figure 5. Dermatofibroma with pale centre and peripheral pigment 
3. Australian Cancer Network Melanoma Guidelines Revision Working Party. Clinical practice guidelines for the management of melanoma in Australia and New Zealand.

Wellington: New Zealand Guidelines Group,

2008. Available at https://melanomapatients. org.au/wp-content/uploads/2017/05/cp111.pdf

[Accessed 2 May 2019].

4. Royal Australian College of General Practitioners. Guidelines for preventive activities in general practice, 9th edn. East Melbourne: Vic: RACGP, 2018. Available at www.racgp.org.au/FSDEDEV/ media/documents/Clinical\%20Resources/ Guidelines/Red\%20Book/Guidelines-forpreventive-activities-in-general-practice.pdf [Accessed 10 April 2019]. 